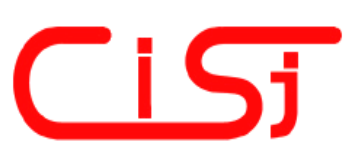

\title{
MOBILE ROBOT LOCALIZATION USING WLAN, ODOMETRY AND GYROSCOPE DATA
}

\author{
Julian Lategahn, Frank Kuenemund, Christof Roehrig
}

\author{
University of Applied Sciences \\ Dortmund, Emil-Figge-Str. 42, \\ 44227 Dortmund, Germany \\ roehrig@ieee.org
}

\begin{abstract}
In this paper a method for estimation of position and motion of a mobile robot in an indoor environment is introduced. The proposed method uses WLAN signal strength to estimate the global position of a mobile robot in an office building. Thus signal strengths of the received access points are stored in the radio map in calibration phase. In localization phase the stored values are compared with actually measured one's. Therefore a fingerprinting algorithm, that was introduced before, is used. The improvement of the presented work is the multi sensor fusion using Kalman filter, which enhances the accuracy of fingerprinting algorithms and tracking of the robot. For this reason odometric and gyroscopic sensors of the robot are fused with the estimated position of the fingerprinting algorithm. The paper presents the experimental results of measurements made in an office building.
\end{abstract}

Keywords: Mobile robots, global localization, Kalman filter, sensor fusion, pose estimation, WLAN, received signal strength.

\section{INTRODUCTION}

Navigation is a key ability of mobile robots. The task of navigation can be divided into localization and path planning. The aim of localization is to estimate the position of a mobile robot in its environment, given a map of the environment and local sensorial data. Robot localization has been recognized as one of the most fundamental problems in mobile robotics.

Aim of localization is to estimate the position of a mobile robot with respect to its environment. The problem is called global localization if there is no priori estimate of the robot position.

Usually odometric sensors are used to solve the localization problem of mobile robots. Odometric sensors provide information about robot movements, but unfortunately, the provided information is noisy and accumulates errors over time in length and orientation. Furthermore with odometry its only possible to get a local position in relation to an initial position. Odometry is accurate enough for local movements but is not suitable for long term localization because of its increasing error [1]. One Method to get those informations is to use a wheel encoder. This kind of encoder is a very low priced sensor and it is available on nearly every wheeled mobile robot.
Because of the insufficient odometry accuracy in the orientation it is helpful to take an other sensor and fuse it with the odometry. A good alternative for this application is a gyroscope. With a gyroscope it is possible to measure the orientation of the mobile robot. Gyroscopes are available in a wide price range from a few dollars to a several thousand dollars. Actual Micro-Electro-Mechanical System (MEMS) gyroscopes are cheap and mainly accurate enough for the localization of a mobile robot. But, as the wheel encoders, its only feasible to get the local orientation of the robot.

Several techniques have been proposed to improve the localization accuracy of mobile robots. A second point in relation to mobile robots is, that they need a sensor to get a global view to their environment, such as a laser scanner or GPS. In this paper the odometric and gyroscopic sensors of a mobile robot are combined with a WLAN localization algorithm [2]. Most WLAN adapters are able to measure the signal strength of received packets as part of their standard operation. The signal strengths of received packets vary noticeably by changing the position [3]. So it can be used to estimate a global position of a mobile robot. To improve the accuracy and the robustness of the position estimation the robot can be tracked by odometric sensors. For more accuracy the position 
calculated by the WLAN signal strength and the odometric position are fused [4]. The sensor fusion is calculated by Kalman filter. The recursive filter calculates out of a series of noisy measurements the position by minimzing the mean of the squared error. The developed system improves the position estimation of a mobile robot as a cheap technology. The costs of mobile robots can be reduced by omitting expensive laser or vision sensors. Also the low computational overhead is an advantage of using Kalman filter for sensor fusion.

This paper is organized as follows:

Section II introduces several kinds of localization techniques in wireless networks.

Section III describes the multi sensor data fusion using the Kalman filter. Therefore the odometric and gyroscope sensors of the mobile robot are combined with the WLAN position estimation. In section IV the used software architecture is presented. Section $\mathrm{V}$ shows the experimental setup of the used robot and the office floor that is used for localization and tracking. The experimental results of the measurements are presented in section VI. Section VII concludes the paper with the evaluation of the levied results and gives a forecast of the future work.

\section{RELATED WORK}

Up to now several kinds of localization techniques are developed for the use in wireless networks. A review of existing techniques is given in [5]. These techniques can be classified by the information they use:

- Connectivity information

- Angle of Arrival (AoA),

- Time of Arrival (ToA),

- Round-trip Time of Flight (RToF),

- Time Difference of Arrival (TDoA),

- Received Signal Strength (RSS).

Connectivity information is available in all kinds of wireless networks. The accuracy of localization depends on the range of the used technology and the density of the beacons. In cellular networks Cell-ID is a simple localization method based on cell sector information [6]. In infrastructure mode of a Wireless LAN (WLAN), the access point (AP) to which the mobile device is currently connected, can be determined since mobile devices know the MAC hardware address of the AP, which they are connected to. Bluetooth is another technology, which allows a relatively accurate localization because of its low radio range [7]. Besides the deployment of Radio Frequency Identification (RFID) in Supply Chain Management [8], the RFID technology is also suitable for position estimation. RFID tags can be deployed at known positions in the environment, in order to obtain position information when they are in range. This information can be fused with data from other sensors (e.g. odometers) for the purpose of improving the accuracy of localization [9][10], such as it is proposed for WLAN in this paper. The high effort and costs for placing RFID tags in a high density makes this technique unfavorable for the indoor localization of a mobile robot.

AoA determines the position with the angle of arrival from fixed anchor nodes using triangulation. In [11] a method is proposed, where a sensor node localizes itself by measuring the angle to three or more beacon signals. Each signal consists of a continuous narrow directional beam, that rotates with a constant angular speed. Drawback of AoA based methods is the need for a special and expensive antenna configuration e.g. antenna arrays or rotating beam antennas.

ToA, RToF and TDoA estimate the range to a sender by measuring the signal propagation delay. The Cricket localization system [12] developed at MIT utilizes a radio signal and a ultrasound signal for position estimation based on trilateration. TDoA of these two signals are measured in order to estimate the distance between two nodes. This technique can be used to track the position of a mobile robot [13]. ToA, RToF as well as TDoA require a complex wireless network infrastructure, which is usually not present in today's WLAN installations. Unfortunately this method requires additional sensors and is not suitable for the target application, which requires cheap technology. UltraWideband (UWB) offers a high potential for range measurement using ToA, because the large bandwidth (> $500 \mathrm{MHz}$ ) provides a high ranging accuracy [14]. In [15] UWB range measurements are proposed for tracking a vehicle in a warehouse. The Ubisense system, developed at the University of Cambridge, is a commercial UWB based localization system [16]. Position estimation is performed using both TDoA and AoA measurements. The anchor nodes are equipped with antenna arrays in order to provide AoA measurements. The TDoA information is determined between pairs of anchor nodes connected with a timing cable. The combination of AoA and TDoA measurement allows a reliable position estimation with an guaranteed accuracy of $15 \mathrm{~cm}$, even if only two anchor nodes receive the signal. Owing to the complex technology, the aboce metioned location system are too expensive. ToA, RToF as wells as TDoA require a complex wireless network infrastructure, which is usually not present in today's WLAN installations. For that reasons these technologies are not applicable for the target application. RSS information can be used in most wireless technologies, since mobile devices are able 
to monitor the RSS as part of their standard operation. The distance between sender and receiver can be obtained with the Log Distance Path Loss Model described in [17]. Unfortunately, the propagation model is sensitive to disturbances such as reflection, diffraction and multipath effects. The signal propagation depends on building dimensions, obstructions, partitioning materials and surrounding moving objects. Own measurements show, that these disturbances make the use of a propagation model for accurate localization in an indoor environment almost impossible [18].

A method to overcome this disadvantage is fingerprinting, which is introduced in [19] and uses a radio map. Fingerprinting is divided in two phases: In the initial calibration phase, the radio map is built by moving around and storing RSS values at various predefined points of the environment. Figure 1 shows an example for a radiomap and its associated distribution of the RSS values in a normal university builing.

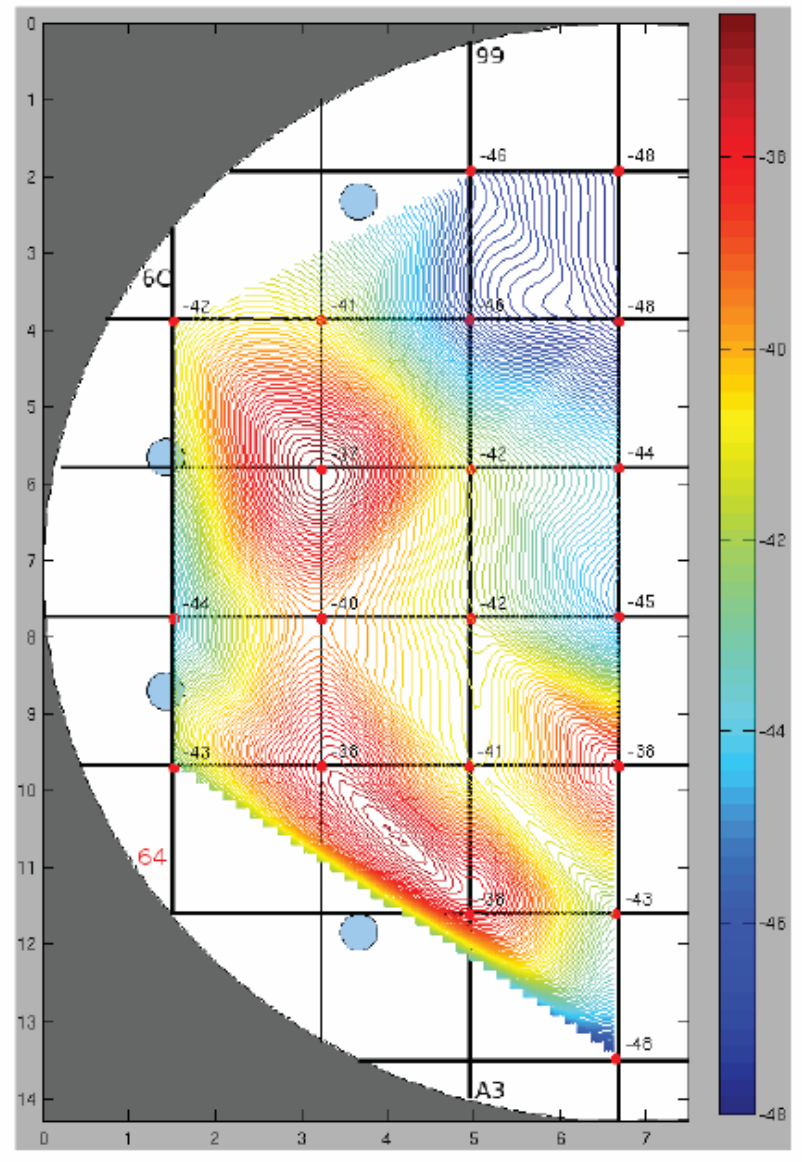

Fig. 1 - Example for a radio map

In the localization phase, the mobile device moves in the same environment and the position is estimated by comparing the current RSS values with the radio map. A metric to compare the measured RSS values with the radio map is Euclidean distance proposed in [19]. Other approaches use a Bayesian algorithm [20] or Delaunay triangulation with lines of constant signal strength [21]. In [22] a Kalman filtering application to an artificial neural network is proposed. An online learning method for radio maps, based on self organizing maps and probabilistic localization algorithm is introduced in [23]. Therefore a rough initial signal propagation model is used to reduce the amount of work in calibration phase. Another approach is presented in [24], where the anisotropy of the antenna gain is exploited to determine heading and integrity of the position, estimated by measured signal strength, of a mobile robot.

\section{MULTI SENSOR FUSION USING KALMAN FILTER}

The Kalman filter was first described by RudolfEmil Kalman in the year 1960. Normaly it is used for multi temporal data fusion of a linear discretetime controlled process but it can be extendend. So it can be used for multi temporal data and multi sensor data fusion. In this extension the structure of the filter stays the same. The filter is divided into two parts, the time update and the measurement update. The time update equations are the following [25]:

$$
\begin{aligned}
& \hat{x}_{k}^{-}=A \hat{x}_{k-1}+B u_{k-1} \\
& P_{k}^{-}=A P_{k-1} A^{T}+Q
\end{aligned}
$$

The time update equations projecting forward the current state $\hat{x}_{k}^{-}$and error covariance estimates $P_{k-1}$ to obtain the a priori estimates for the next time step. The measurement update can be described as corrector equations:

$$
\begin{aligned}
& K_{k}=P_{k}^{-} H^{T}\left(H P_{k}^{-} H^{T}+R\right)^{-1}, \\
& \hat{x}_{k}=\hat{x}_{k}^{-}+K_{k}\left(z_{k}-H \hat{x}_{k}^{-}\right), \\
& P_{k}=\left(I-K_{k} H\right) P_{K}^{-},
\end{aligned}
$$

where $K_{k}$ is the Kalman gain, which is used in (4) for weigthing the difference between the a priori estimate and the current actually measure $z_{k}$. Then with (4) the $a$ posteriori state estimate is calculated with the a priori state estimate and by the Kalman gain weighted difference. With (5) the a posteriori error covariance is estimated. After each pair of update equations the previous a posteriori estimates are used to predict the new a priori estimates. The recursive nature of the Kalman filter makes it attractive for a wide field of applications. For the fusion every measurement of the appropriate sensor at the time $k$ is combined in an extendended measure 
vector $\quad z_{k}=\left(\left(z_{k}{ }^{(1)}\right)^{T},\left(z_{k}{ }^{(2)}\right)^{T}, \ldots,\left(z_{k}{ }^{(M)}\right)^{T}\right)^{T}$. The system is defined in $M$ equations [26]:

$$
x_{k}=A x_{k-1}+B u_{k-1}+w_{k-1}
$$

with the process noise

$$
p\left(w_{k}\right) \sim N\left(0, Q_{k}\right)
$$

The equation shows that the process noise is independent, white and with a normal probability distribution. The process noise covariance $Q_{k}$ is important to the behaviour of the Kalman filter. The values for $Q_{k}$ declares how to trust the process. The equations 6 and 7 are similar to the Kalman filter without fusion. The differences are only located in the measurement equations. The measurements of the respective sensors $S^{(m)}$ are described as follows:

$$
Z_{k}^{(m)}=H^{(m)} X_{k}+V_{k}^{(m)}
$$

with the measurement noise

$$
p\left(v_{k}^{(m)}\right)-N\left(0, R_{k}^{(m)}\right)
$$

Also the measurement noise is independent, white and with normal probability distribution. As the process noise covariance $Q_{k}$, it is necessary to find a matching value for the measurement noise covariance $R_{k}^{(m)}$. With this matrix it is possible to specify how the different sensors $S^{(m)}$ are trusted. The measurement noise covariance $R_{k}^{(m)}$ is built from the covariances of each sensor:

$$
R_{k}=\left(\begin{array}{ccc}
R_{k}^{(1)} & 0 & 0 \\
0 & \ddots & 0 \\
0 & 0 & \left.R_{k}^{(M)}\right)
\end{array}\right)
$$

Furthermore the matrix $H^{(m)}$ is composed from different gain matrices related to the associated sensor $S^{(m)}$ :

$$
H=\left(\begin{array}{c}
H^{(1)} \\
\vdots \\
H^{(M)} 0
\end{array}\right)
$$

The archtitecture of the measurement fusion is centralized, that means that all measurements of each sensor are collected together at a central point. The Fig. 2 shows the centralized measurement fusion for a mobile robot, where $z_{w}$ is the position estimated out of the WLAN signal strength and $z_{O}$ the position calculated with odometry and gyroscope data. Note that $x_{w}$ and $x_{o}$ are the $x$ positions of the WLAN localization and the odometry and does not deal with the state $x_{k}$.

The state of the concrete system $x_{k}$ consits, as shown in equation 12, of the position $x$ an the velocity in $x$ direction $v_{x}$.

$$
x_{k}=\left(\begin{array}{c}
x \\
v_{x}
\end{array}\right)
$$

$y$ and $v_{y}$ are not part of this filter. Because of the performance this system is splitted into two filters for $x$ and $y$. But the design for both filter is equal, so here is only one direction introduced. The matrix $A$ predict the new position across the velocity as follows:

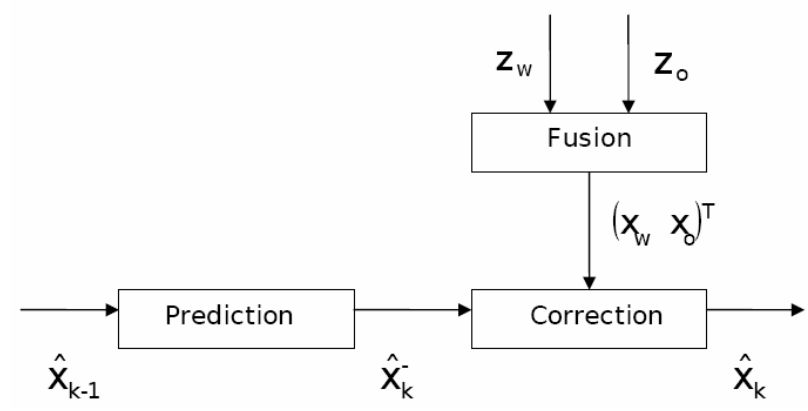

Fig. 2 - Centralized measurement fusion [26]

$$
A=\left(\begin{array}{cc}
1 & \Delta t \\
0 & 1
\end{array}\right)
$$

The values for the process noise covariance $Q$ are identified empiricaly. As shown in equation 14 an identity matrix was choosen for $Q$ :

$$
Q=\left(\begin{array}{ll}
1 & 0 \\
0 & 1
\end{array}\right)
$$

The matrix is equal in every sampling step so $k$ could be omitted for the process noise covariance $Q$. The matrices $H^{w}$ and $H^{o}$ are composed to a new gain matrix $H$ and is set as follows:

$$
H=\left(\begin{array}{ll}
1 & 0 \\
1 & 0
\end{array}\right) .
$$


The measurement vector $z_{k}$ consits of the $x$ position $x_{w}$ computed of the actual WLAN scan and the position $x_{o}$ computed of the wheel encoder. $z_{k}$ is shown in equation 16 :

$$
z_{k}=\left(\begin{array}{c}
x_{w} \\
x_{o}
\end{array}\right)
$$

Once again as the process noise covariance $Q$ the sampling step $k$ could be omitted for the measurement noise covariance $R$, because there is no change in it. $R$ is composed of $R_{w}$ and $R_{o}$ and shown in equation 17 :

$$
R=\left(\begin{array}{cc}
R_{w} & 0 \\
1 & R_{o}
\end{array}\right)=\left(\begin{array}{cc}
5 & 0 \\
0 & 0,2
\end{array}\right)
$$

The variances for the several entries of the measurement noise covariance $R$ can be measured previously by an offne application. The result of these analysis were that the odometry position has a much lower variance than the WLAN position. So the value for $R_{w}$ is set to 5 and much higher than the value for $R_{o}$ with 0,2 .

The odometry and gyroscope data are pre filtered with an independant Kalman filter on the microcontroller of the Pioneer3-AT.

The signal strength to estimate the position $x_{W}$ and $y_{W}$ is pre filtered with an independent Kalman filter. The filter matrices are scalar for this kind of system. So the values for $A, H$ and the initial for $P_{0}$ are set to 1 . The initial value for the state $x_{0}$ is set to the first signal strength measurement that is done. As seen in figure 3 the unfiltered signal strength is incorrect.

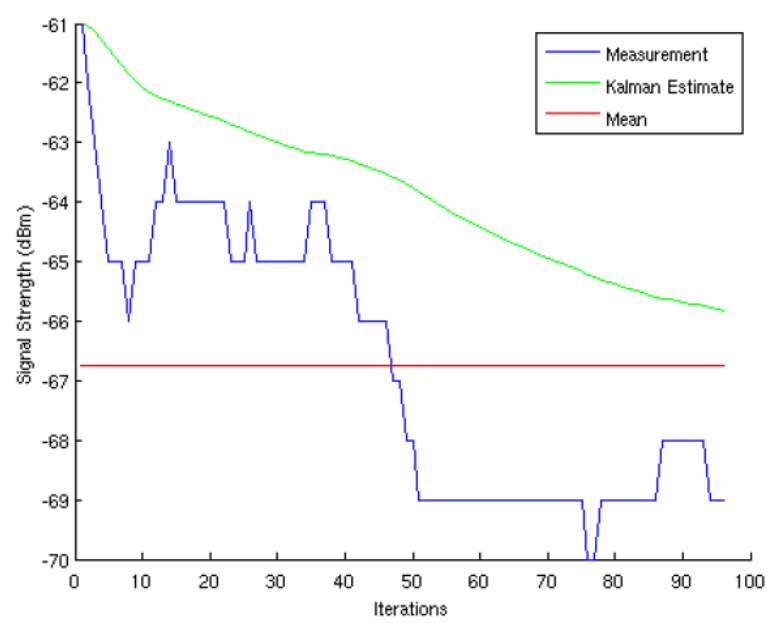

Fig. 3 - Filtered signal strength for a stable position

The curve for the filtered signal shows that it is more smoothed, but it is necessary to distinguish stable positions and while the robot is in motion. For stable positions its better to choose a smaller value for the measurement noise covariance $R_{k}$ and a higher for the process noise covariance $Q_{k}$. The picture 3 shows this behaviour in a situation while the robot does not drive.

If the robot is in motion its the other way around, a higher value for $R_{k}$ and a smaller for Qk provides a better result. As seen in figure 4 the filtered curve is more similar to the measurement than the one in figure 3.

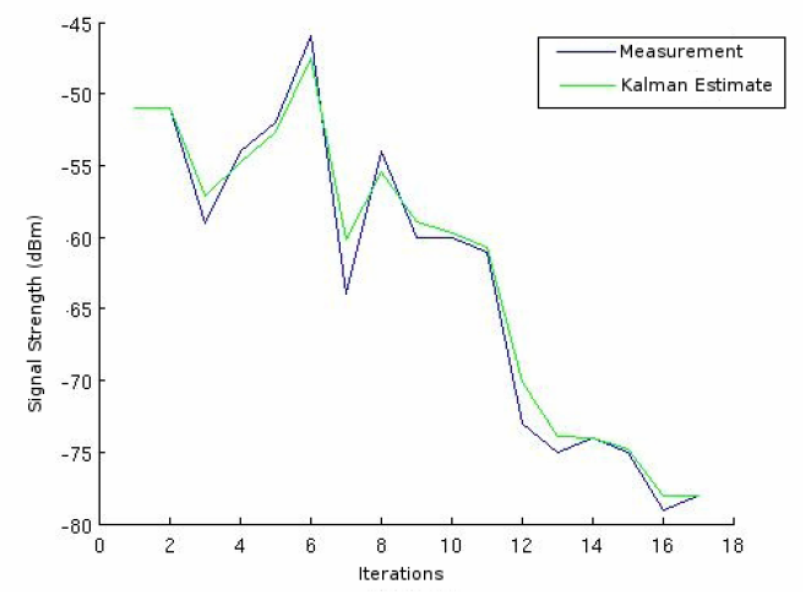

Fig. 4 - Filtered signal strength in motion

\section{SOFTWARE DESIGN}

The software is divided into three parts: a localization engine, a graphical user interface (GUI) and a WLAN scanner (Fig. 5). The localization engine and the GUI are written in the Matlab script language, the WLAN scanner are implemented in C. The WLAN scanner uses the WE ioctl()Interface for reading RSS values from both WLAN adapters.

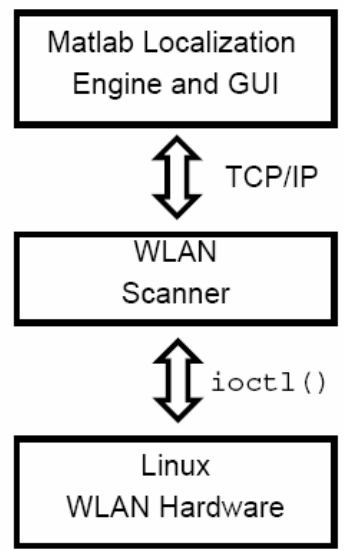

Fig. 5 - Design of the localization software

The communication between localization engine and WLAN scanner is build with TCP/IP sockets. Since the localization engine are built on Matlab, it is possible to run it on every computer which offers a Matlab environment and a network access. The GUI is used for monitoring information to the user 
and for building the radio map. Fig. 6 shows the GUI with a map of a floor in the Computer Science building. The red dots show the measured referenced robotics tasks such as sensor fusion, localization, mapping, and navigation. For programming purposes ActivMedia provides the toolkit ARIA

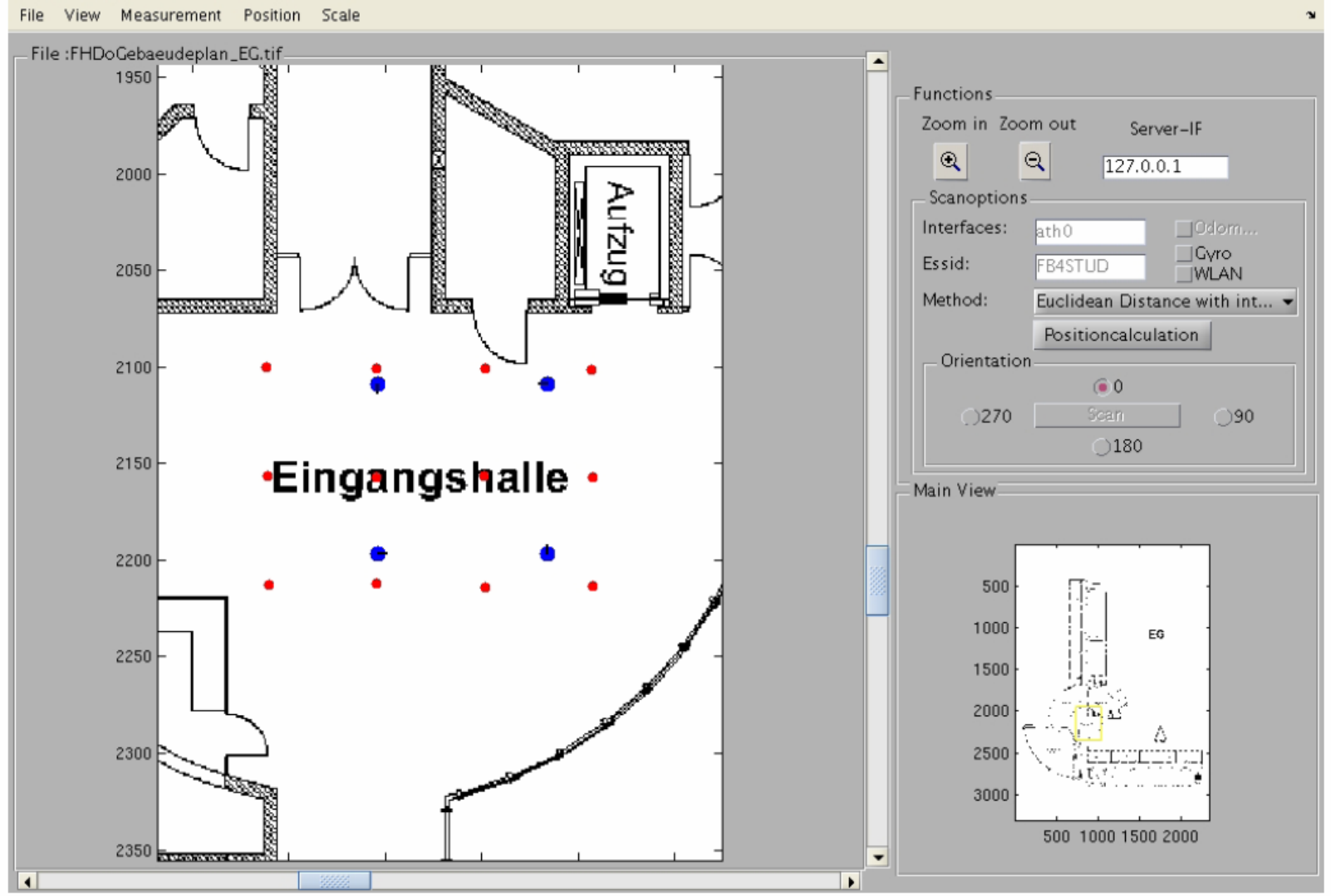

Fig. 6 - Matlab Localization engine and GUI

points that were learned in the calibartion phase. In order to build the radio map, the user moves the robot to the predefined poses (red arrows) and stores the RSS values. It is optional to change the Server IP address, the network interfaces for both antennas and the ESSID of the APs. The blue circles define the trajectory the robot moves when the examination is conducted. The heading of the robot is shown with black line on the blue circles. Deviation of the real heading of the robot is measured after every examination in the final position (see $\Delta \Theta$ in table 1 ).

\section{EXPERIMENTAL SETUP}

The experiments are carried out with a mobile robot Pioneer3-AT manufactured by MobileRobots Inc. (Fig. 7). The robot is equipped with an embedded computer for real time robot control and an additional PC with a WLAN card for communication and localization.

A robot server is included in the operating system of the embedded computer. It manages the low-level tasks of robot control and operation, including motion and odometry. The robot server receives the commands from the PC via RS-232 serial link. It is the job of a program running on the PC to perform
(ActivMedia Robotics Interface for Application)

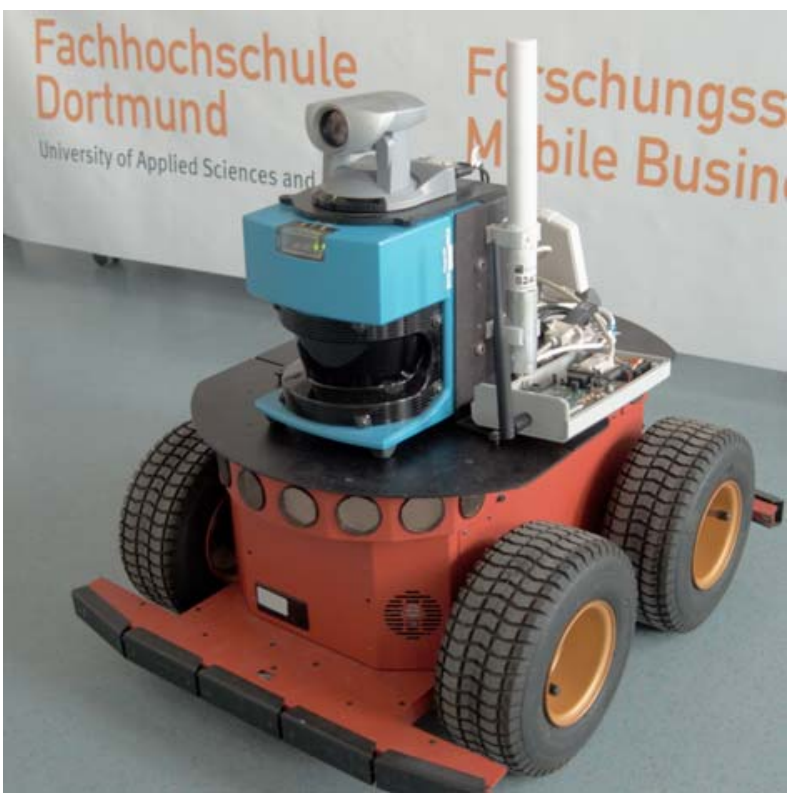

Fig. 7 - Pioneer3-AT

[27]. ARIA is a object oriented, cross-platform (Windows /Linux) toolkit for ActivMedia mobile robots. It is written entirely in $\mathrm{C}++$, but access to the API is also available from the Java programming languages via "wrapper" libraries. ARIA provides an 
interface to control the robot's velocity, heading, relative heading, and provide detailed information about odometry and operating conditions from the mobile robot.

The operation system on the PC is Debian Linux (5.0 Lenny), which offers support for wireless communication by the wireless extension (WE). WE is an application programming interface (API) allowing a user space program to configure a WLAN driver and receive statistic information. The WE provide an interface via ioctl(), which is documented in wireless.h. Good examples for programming WE are the wireless tools for Linux. The program iwlist scans the WLAN for accessible APs and monitors the signal strengths of received packets along with hardware MAC addresses of APs in range.

There are two kinds of scanning modes: passive scanning and active scanning. In passive scanning mode the WLAN card is put into monitoring mode and waits for incoming packets. In active scanning mode the mobile device sends a probe request packet on every frequency and waits for the probe response packets of the access points (APs) in range. Active scanning is an important feature in WLAN positioning, because the time of the measurements for all received signal strengths can be determined. Active scanning obtains new received signal strength values of all APs in range at the time of scanning.

\section{EXPERIMENTAL RESULTS}

The experiments were carried out in a foyer of an unversity building. The mobile robot was driven around a square with $3 \mathrm{~m}$ side length. The measured points of the WLAN signal strength were made in an equal distance of $2 \mathrm{~m}$ and stored into the radio map. At each time of the measurements, five access points of the facultys internal WLAN were reachable. Four tests with different sensor fusions were conducted. Although by the first two experiments the robot was driven one round and by the third and fourth experiment two rounds. The table I shows the experimental results, where _p is the error of the position and _ of the heading of the robot. As shown in the table I the fusion of sensor data from WLAN, odometry and gyroscope provides the best results. The greater deflection in the second round can be explained with the accumulated error of the odometric sensors. The improvement of using sensor fusion would be even better with a larger terrain. In conclusion the sensor fusion with the estimated WLAN position is an advantage, when the wheel encoders deliver position failures of 1 to $2 \mathrm{~m}$ to the real position of the robot.
Table 1. Error of the estimated positions

\begin{tabular}{|c|c|c|c|c|}
\hline \multirow{2}{*}{} & \multicolumn{4}{|c|}{1 Round } \\
\cline { 2 - 5 } & \multicolumn{2}{|c|}{ Test 1} & \multicolumn{2}{c|}{ Test 2} \\
\cline { 2 - 5 } & $\Delta p$ & $\Delta \Theta$ & $\Delta p$ & $\Delta \Theta$ \\
\hline Odo & $0,62 \mathrm{~m}$ & 13 & $0,35 \mathrm{~m}$ & 6 \\
\hline Odo/WLAN & $0,51 \mathrm{~m}$ & 13 & $0,26 \mathrm{~m}$ & 6 \\
\hline Odo/Gyro & $0,12 \mathrm{~m}$ & 1 & $0,24 \mathrm{~m}$ & 8 \\
\hline Odo/Gyro/WLAN & $0,12 \mathrm{~m}$ & 1 & $0,14 \mathrm{~m}$ & 8 \\
\hline WLAN & $1,42 \mathrm{~m}$ & - & $2 \mathrm{~m}$ & - \\
\hline & \multicolumn{4}{|c|}{1 Round } \\
\cline { 2 - 5 } & \multicolumn{2}{|c|}{ Test 1} & \multicolumn{2}{c|}{ Test 2} \\
\cline { 2 - 5 } & $\Delta p$ & $\Delta \Theta$ & $\Delta p$ & $\Delta \Theta$ \\
\hline Odo & $1 \mathrm{~m}$ & 32 & $0,85 \mathrm{~m}$ & 20 \\
\hline Odo/WLAN & $0,92 \mathrm{~m}$ & 32 & $0,8 \mathrm{~m}$ & 20 \\
\hline Odo/Gyro & $0,28 \mathrm{~m}$ & 13 & $0,34 \mathrm{~m}$ & 6 \\
\hline Odo/Gyro/WLAN & $0,29 \mathrm{~m}$ & 13 & $0,3 \mathrm{~m}$ & 6 \\
\hline WLAN & $1,13 \mathrm{~m}$ & - & $0,7 \mathrm{~m}$ & - \\
\hline
\end{tabular}

\section{CONCLUSION AND FUTURE WORKS}

This paper has presented a method for the fusion of multi sensor data of a mobile robot. Therefore a Kalman filter is applied to the position estimated by a fingerprinting algorithm and the measurements of the odometry and gyroscope sensors. In future work the Kalman filter for fusing the wheel encoders and the gyroscope done by the Pioneer3-AT would be replaced by an own implementation to have a better access to this point of fusion. Another Kalman filter is applied to reduce the noise of the signal strength measurements. The results show that the fusion of all three sensory data provides the best accuracies of the estimated positions and the tracking of the mobile robot. The improvement of the sensor data fusion using Kalman filter would be even more noticeable driving a longer trajectory. For this examination a larger terrain is needed, what will be done in future work.

Furthermore in future work it is intended to use an online learning algorithm to enhance the radio map. Also the adaption on the temporal measured signal strengths could be realized. For this purpose a method based on the self organizing maps from Theuvo Kohonen [28] seems to be the best approach.

\section{REFERENCES}

[1] S. Thrun, Probabilistic Algorithms in Robotics, AI Magazine, 2000, vol. 21, no. 4, pp. 93-109.

[2] C. Röhrig and F. Künemund, WLAN based Pose Estimation for Mobile Robots, in Proceedings of the 17th World Congress The International Federation of Automatic Control, Seoul, Korea, July 2008.

[3] I. Adusei, S. Kopetzki, and B. Goebel, Developing a Real Time RSSI-based Mobile Positioning System," in Proceedings of the 1st 
Workshop on Positioning, Navigation and Communication, 2004, pp. 119-122.

[4] J. Lategahn, WLAN-Lokalisierung eines mobilen Roboters mit Sensordatenfusion, University of Applied Sciences and Arts, Dortmund, Diploma Thesis, 2008.

[5] M. Vossiek, L. Wiebking, P. Gulden, J. Wieghardt, C. Hoffmann, and P. Heide, Wireless Local Positioning, Microwave Magazine, Dec. 2003, vol. 4, no. 4, pp. 77-86.

[6] Guolin Sun, Jie Chen, Wei Guo, and K. Liu, Signal Processing Techniques in Networkaided Positioning: A Survey of State-of-the-art Positioning Designs, Signal Processing Magazine, July 2005, vol. 22, no. 4, pp. 12-23.

[7] S. Feldmann, T. Hartmann, and K. Kyamakya, An indoor Bluetooth-based Positioning System: Concept, Implementation and Experimental Evaluation, in Proceedings of the International Conference on Wireless Networks, Las Vegas, USA, June 2003, pp. 109-113.

[8] K. Michael and L. McCathie, The Pros and Cons of RFID in Supply Chain Management, in Proceedings of the International Conference on Mobile Business, Sydney, Australia, July 2005, pp. 623-629.

[9] D. Hahnel, W. Burgard, D. Fox, K. Fishkin, and M. Philipose, Mapping and Localization with RFID Technology, in Proceedings of the International Conference on Robotics and Automation, vol. 1, New Orleans, USA, May 2004, pp. 1015-1020.

[10] J. Koch, J. Wettach, and E. Bloch, Indoor Localisation of Humans, Objects, and Mobile Robots with RFID Infrastructure, in Proceedings of the 7th International Conference on Hybrid Intelligent Systems, Kaiserslautern, Germany, Sept. 2007, pp. 271276.

[11] A. Nasipuri and K. Li, A Directionality based Location Discovery Scheme for Wireless Sensor Networks, in Proceedings of the 1st ACM International Workshop on Wireless Sensor Networks and Applications, Atlanta, USA, Sept. 2002, pp. 105-111.

[12] N.B. Priyantha, A.K.L. Miu, H. Balakrishnan, and S. Teller, The Cricket Compass for Context-aware Mobile Applications, in Proceedings of the 7th Annual International Conference on Mobile Computing and Networking, Rome, Italy, July 2001, pp. 1-14.

[13] P. Alriksson and A. Rantzer, Experimental Evaluation of a Distributed Kalman Filter Algorithm, in Proceedings of the 46th IEEE Conference on Decision and Control, New Orleans, Dec. 2007, pp. 5499-5504.

[14] S. Gezici, Zhi Tian, G. Giannakis,
H. Kobayashi, A. Molisch, H. Poor, and Z. Sahinoglu, Localization via Ultra-wideband Radios: A Look at Positioning Aspects for Future Sensor Networks, Signal Processing Magazine, July 2005, vol. 22, no. 4, pp. 70-84.

[15] J. Fernandez-Madrigal, E. Cruz, J. Gonzalez, C. Galindo, and J. Blanco, Application of UWB and GPS Technologies for Vehicle Localization in Combined Indoor-Outdoor Environments, in Proceedings of the International Symposium on Signal Processing and its Applications, Sharja, United Arab Emirates, Feb. 2007.

[16] J. Cadman, Deploying Commercial Locationaware Systems," in Proceedings of the 2003 Workshop on Location-Aware Computing, Seattle, USA, Oct. 2003, pp. 4-6.

[17] N. Patwari, A.O. Hero, M. Perkins, N.S. Correal, and R. O’Dea, Relative Location Estimation in Wireless Sensor Networks, IEEE Transactions on Signal Processing, 2003, vol. 51, no. 8, pp. 2137-2148.

[18] C. Röhrig and F. Künemund, Estimation of Position and Orientation of Mobile Systems in a Wireless LAN," in Proceedings of the 46th IEEE Conference on Decision and Control, New Orleans, USA, Dec. 2007, pp. 4932-4937.

[19] P. Bahl and V.N. Padmanabhan, RADAR: An In-Building RFbased User Location and Tracking System, in Proceedings of the 19th Annual Joint Conference of the IEEE Computer and Communications Societies, vol. 2, Tel Aviv, Israel, Mar. 2000, pp. 775-784.

[20] A.M. Ladd, K.E. Bekris, A. Rudys, D.S. Wallach, and L.E. Kavraki, On the Feasibility of Using Wireless Ethernet for Indoor Localization, IEEE Transaction on Robotics and Automation, June 2004, vol. 20, no. 3, pp. 555-559.

[21] U. Großmann, C. Röhrig, S. Hakobyan, T. Domin, and M. Dalhaus, WLAN Indoor Positioning based on Euclidian Distance and Interpolation (Isobars), in Proceedings of the 8th Wireless Technologies Kongress, Dortmund, Germany, 2006, pp. 296-305.

[22] S. Outemzabet and C. Nerguizian, Accuracy enhancement of an indoor ann-based fingerprinting location system using Kalman filtering, in IEEE 19th International Symposium on Personal, Indoor and Mobile Radio Communications, Sept. 2008, pp. 1-5.

[23] B.B. Parodi, A. Szabo, J. Bamberger, and J. Horn, "Spll: Simultaneous probabilistic localization and learning," in Proceedings of the 17th World Congress on The International Federation of Automatic Control, Seoul. Korea, July 2008.

[24] J. Graefenstein, A. Albert, and P. Biber, 
Radiation pattern correlation for mobile robot localization in low power wireless networks, in Proceedings of the 2009 IEEE International Conference on Robotics and Automation, 2009.

[25] G. Welch and G. Bishop, An introduction to the Kalman filter, Department of Computer Science University of North Carolina at Chapel Hill, Tech. Rep., July 2006.

[26] H.B. Mitchell, Multi-Sensor Data Fusion: An Introduction, Springer, 2007.

[27] A. Robotics, Advanced Robotics Interface for Applications (ARIA) Developer's Reference Manual, ActivMedia Robotics, LLC., 2006, http://robots.activmedia.com/ARIA.

[28] T. Kohonen, The self-organizing map, Proceedings of the IEEE, Sep 1990, vol. 78, no. 9, pp. 1464-1480.

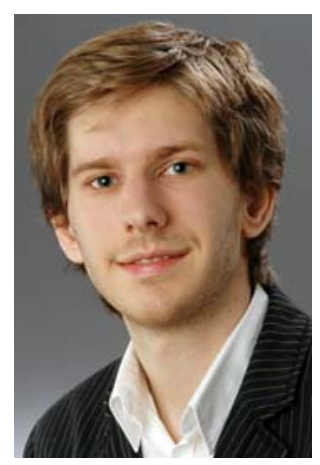

Julian Lategahn received his Diploma degree from the University of Applied Sciences Dortmund, Germany, in 2008, in computer science. After his degree he works in a scientific project at the University of Applied Sciences Dortmund. Furthermore he currently is studying for his master's degree in computer science at the University of Applied Sciences in Dortmund. His current research interests include sensor data fusion, kalman filter and localization.

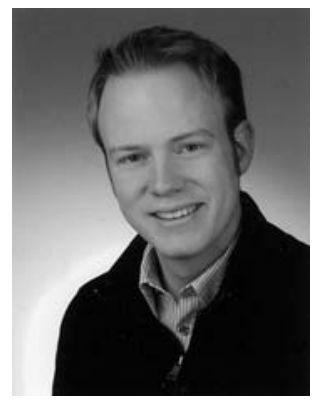

Frank Künemund received his Diploma degree from the University of Applied Sciences Dortmund, Germany, in 2008, in computer science. After his degree he works in the scientific staff of the University of Applied Sciences Dortmund. Furthermore he is a student of master of computer science degree course of the University of Applied Sciences Dortmund. His current research interests include robotics, localization and WLAN technologies.

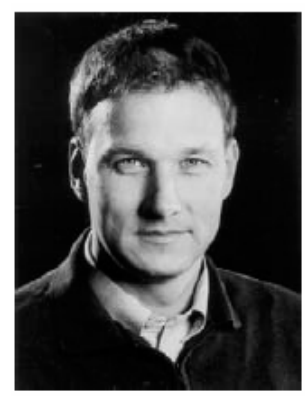

Christof Röhrig received his Diploma degree from the University of Bochum, Germany, in 1993, and his Doctor degree from the University of Hagen, Germany, in 2003, both in electrical engineering. Between 1993 and 1997 he was Manager Automated Systems

Engineering at Reinoldus Transport- und Robotertechnik GmbH Dortmund, Germany. From 1997 until 2003 he was with the Control Systems Engineering Group at University of Hagen. His research was focused on motor control, eLearning and remote laboratories. Since 2003, he is Professor of Computer Science at the University of Applied Sciences Dortmund, Germany. His current research interests include robotics, mobile computing and telematics. 